\title{
Medium-term coordination in a network-constrained multi-period auction model for day-ahead markets of hydrothermal systems
}

\author{
Gabriela Fernanda Bregadioli a , Edméa Cássia Baptista ${ }^{\mathrm{b}}$, Leonardo Nepomuceno ${ }^{\mathrm{a}, *}$, \\ Antonio Roberto Balbo ${ }^{\mathrm{b}}$, Edilaine Martins Soler ${ }^{\mathrm{b}}$ \\ a Department of Electrical Engineering, Faculty of Engineering-FEB, Unesp-Univ. Estadual Paulista, 17033-360 Bauru, SP, Brazil \\ ${ }^{\mathrm{b}}$ Department of Mathematics, Faculty of Sciences-FC, Unesp-Univ. Estadual Paulista, 17033-360 Bauru, SP, Brazil
}

\section{A R T I C L E I N F O}

\section{Article history:}

Received 1 June 2015

Accepted 20 March 2016

Available online 18 April 2016

\section{Keywords:}

Energy markets

Short-term generation scheduling

Coordination strategies

Auction models

Hydrothermal systems

\begin{abstract}
A B S T R A C T
This paper investigates strategies for coordinating the decisions calculated by a network-constrained multi-period auction model of hydrothermal systems with the ones calculated by medium-term problems. Initially, we discuss the necessity for implementing such coordination strategies in hydrothermal systems from the standpoint of a market operator. We investigate three alternative strategies for performing the coordination. The first strategy incorporates primal information from the medium-term problem into the auction model in order to perform coordination, while the second one makes use of dual information associated with the medium-term. A third strategy combining primal and dual information from the medium-term problem is also investigated. These strategies are compared by means of simulation results involving the IEEE 24-bus reliability test-system. The results focuses on evaluating the impact of the coordination strategies on market clearing prices and generation scheduling.
\end{abstract}

(c) 2016 Elsevier Ltd. All rights reserved.

\section{Introduction}

In the regulated environment, the short-term scheduling of power generation systems was generally carried out by means of a centralized procedure run by the system operator. The main purpose behind the models used to calculate such a centralized dispatch was to minimize the total costs associated with the generation system, while meeting a specific inelastic demand and enforcing all relevant operational constraints associated with the system. For such a purpose, unit commitment (UC) models $[13,18]$ were generally used for thermal systems, while for hydro-dominated systems, short-term hydrothermal scheduling (STHS) [17] or predispatch models [25] were generally used. In such a context, the coordination of short- and long-term generation has been performed by means of a chain of models associated with long, medium and short-term scheduling [19,21].

In the electricity markets environment, short-term scheduling procedures were reoriented in order to promote competition. The old centrally dispatched short-term scheduling procedures were

* Corresponding author at: Av. Eng. Luiz Edmundo C. Coube 14-01, Dept. of Electrical Engineering, 17033-360 Bauru, SP, Brazil. Tel.: +55 1431036115.

E-mail addresses: gaby_fb.dc@hotmail.com (G.F. Bregadioli), baptista@fc.unesp. br (E.C. Baptista), leo@feb.unesp.br (L. Nepomuceno), arbalbo@fc.unesp.br (A.R. Balbo), edilaine@fc.unesp.br (E.M. Soler). replaced by auction procedures [1,15], where the generation and demand compete in the day-ahead market in order to sell and buy energy, respectively. In the auction procedure, blocks of offers and bids, provided respectively by generation companies (GENCOS) and consumers, are submitted to the market operator (MO), which calculates the accepted blocks of offers and bids, by means of a market-clearing procedure, which is formulated as an auction model.

According to Kardakos et al. [12], centrally organized day-ahead markets may take two basic forms: power exchanges (PX) or power pools (PP). In the PX market, offers and bids are handled hour-by-hour by a series of independent single-period auction models run by the MO. These models tend to neglect the technical aspects associated with the generating units, or with the transmission system. In the PX, each producer is responsible for selfscheduling his own units, by a price-based unit commitment $[14,26]$, while the independent system operator (ISO) is responsible for preserving the system security. For such a purpose, the ISO must handle all technical aspects neglected in the auction by means of ex-post heuristic procedures that generally tend to redispatch the system. In the PP market, offers and bids are handled by a multi-period auction model that integrates all technical aspects related to the units (e.g., unit start-up and shut-down costs, minimum-up/down time constraints, min/max power output restrictions, ramp-rate limits, etc.) and the transmission 
system into a single optimization problem. This model is concerned with the maximization of the social welfare function, while taking into account relevant constraints associated with the generation and transmission systems. The model calculates the on/off schedules of the generating units, the active power dispatch of the scheduled units, as well as the market-clearing prices throughout the day.

PX based energy markets are generally adopted for hydropower or hydro-dominated systems [6,27], probably due to its transparency and easiness of implementation and operation. Hydropower systems are generally more complicated to operate due to the need for representing additional modeling aspects associated with the hydraulic system, such as: limits in reservoirs levels and volumes, nonlinear limits in water discharges and power outputs, and uncertainties in future water inflows and prices. Also, these systems must have some sort of coordination strategy in order to properly manage the use of hydro resources in the short- and medium-terms. Such additional modeling issues also contribute to the adoption of PX based energy markets for hydropower systems, since these constraints are handled in an simpler and transparent way in such markets, by means of ex post alterations in dispatch and prices. However, all such ex post alteration may lead to suboptimal operation points in terms of social welfare, as well as to cross-subsidies, as shown by Conejo et al. [2]. On the other hand, a PP based energy market would integrate all such additional constraints into the auction model, avoiding such economic distortions.

This paper investigates a crucial issue concerning the implementation of PP based markets for hydropower systems: the coordination strategies. The main contributions of the paper are: (i) we discuss the need to coordinate short-term decisions, calculated by a hydrothermal day-ahead auction model, with medium-term decisions; (ii) we propose and investigate three alternative coordination schemes for such a purpose; (iii) we propose a network-constrained multi-period hydrothermal auction (MHA) model that incorporates the coordination procedures discussed in (ii) for a pool-based energy market; and (iv) in the proposed MHA model, the thermal constraints are represented in detail, while the transmission system is represented by means of linear power flows and piecewise linear approximation for power losses. Hydro constraints are represented in a simplified way, by means of coordination equations only.

What remains of this work is organized as follows: in Section "Coordination strategies in hydrothermal auction models" we discuss the need to coordinate as well as some coordination strategies in hydrothermal auction models; in Section "Multi-period hydrothermal auction model with medium-term coordination", we describe the network-constrained multi-period hydrothermal auction (MHA) model and three coordination strategies are incorporated in the model. Numerical results evaluating the impact of the coordination strategies studied are described in Section "Numerical results". Finally, the conclusions are presented in Section "Conclusions".

\section{Coordination strategies in hydrothermal auction models}

\section{The need to coordinate}

According to Conejo et al. [3] the market operator (MO) is responsible for the economic management of the electricity marketplace as a whole, while the independent system operator (ISO) is in charge of the technical management of the electric energy system pertaining to the marketplace. In some markets, the functions performed by the MO and ISO are carried out by a single entity. The ISO and the MO must establish sound rules on the electricity markets in order to operate them efficiently while ensuring security and reliability of the power system [23].

In hydro-dominated systems, the concepts of security and reliability are strongly related to the availability of hydro resources. The key economic issue in hydro-power production is time dependency: the water used today can alternatively be stored in reservoirs to be used tomorrow [5]. Therefore, there must be some kind of coordination of hydro resources between short- and medium-term planning. Although each independent generating company is concerned with the coordination of its own hydro resources, it is not reasonable to leave the responsibility of coordinating the hydro resources of the entire system for these companies. Since coordination directly affects security and reliability of hydro-dominated systems, the task of coordinating the hydro resources of the system must be carried out by the ISO.

In a traditional regulated environment, the coordination between different time scopes is developed by a system operator trying to minimize the total cost of the system [22]. Some models addressing this problem are described in Soares et al. [24], Pereira and Pinto [19,20] and Franco et al. [7]. In such approaches, the main idea is to describe short- and medium-term problems by a single large optimization model. Then, by using some decomposition approach (e.g. Benders or Dantzig-Wolfe decomposition techniques), this large model is broken into two sub-models, which are associated with short- and medium-term subproblems, respectively. Therefore, in these approaches, the coordination between medium-term planning and short-term operation could be seen as an iteration in the framework of the decomposition theory for optimization problems [22].

Reneses et al. [22] analyze the problem of coordinating resources between short- and medium-term in an electricity marketplace. The analysis is performed from the perspective of a generating company, trying to maximize its profits in the market. The authors point out that short- and medium-term scheduling models used by a generating company in the market are different in essence. While short-term approaches are formulated as unilateral profit maximization models, medium-term approaches are generally formulated as equilibrium models. Therefore, by analyzing the perspective of a generating company in a marketplace, Reneses et al. [22] conclude that medium and short-term models do not result from the decomposition of a single larger model.

The same reasoning apply if an analysis is performed from the perspective of a MO or an ISO seeking to schedule the system resources (generation and water) in the short- and mediumterms. In this case, the short-term scheduling approaches used by the MO involve market clearing procedures (mathematically described by means of auction models), while the medium-term approaches may also be formulated by means of equilibrium models or by auction models (futures market). Thus, we conclude that from the perspective of a MO or an ISO, the auction (short-term) and the equilibrium models (medium-term) may also not result from the decomposition of a larger optimization problem. However, as pointed out in Reneses et al. [22], in spite of this, we may still use decomposition theory as an inspiration for helping the MO or the ISO in their tasks for coordinating short- and medium-term generation scheduling.

The authors in Reneses et al. [22] highlight some coordinating issues that may appear when a generation company, owning only thermal units, is seeking to maximize its profits in the energy market, such as: (i) the need to establish maximum daily production levels for thermal units with limited medium-term emission allowances (scarce resources) and (ii) the need to fulfill minimum fuel-consumption requirements throughout the day due to a take-or-pay contract or a minimum market share that must be accomplished by the company in the medium-term 
(obligatory-use resources). Note that, if a generation company, owning only thermal units, neglects these coordination issues, this may compromise the performance of such company in the market, but it does not compromise the system security and reliability. Thus, short- and medium-term coordination for thermal systems is not a relevant constraint to be represented by the MO or the ISO in auction models. That is why the coordination schemes are neglected in the auction models proposed for thermal systems. In fact, as far as we know, no other auction model in the literature incorporates coordination strategies. The need to introduce coordination strategies in the auction procedure has not yet been clearly discussed in the literature.

Next, we perform a careful analysis concerning the need to coordinate short-term scheduling (calculated by means of auction models) with medium-term decisions in a hydro-dominated system. This analysis is performed by considering two possible dayahead market structures: a power exchange (PX) or a power pool (PP). For such a purpose, consider a MO seeking to clear the dayahead energy market of a hydro-dominated system by means of an auction model.

If the day-ahead market takes the form of a PX, it is generally cleared in an hourly basis by means of simple auction procedures that neglect most constraints associated with generation and transmission systems, as well as the representation of coordination strategies. In the PX, the basic idea is to keep the market clearing procedure as simple as possible. Therefore, the introduction of coordination strategies in the auction model used by a PX could violate this transparency rule. Also, in the PX, the generation companies are responsible for scheduling their own hydro units, by means of price-based unit commitment procedures. Nevertheless, these individual schedules provided by the companies must be evaluated by the ISO regarding security and reliability, which means that the ISO may alter the generation dispatch in order to comply with security requisites. This ex post alterations in the dispatch are necessary in order to take into account both congestion and losses in the transmission system, which are generally neglected in the simple market clearing procedures of the PX. In hydro-dominated systems, the ISO must consider an additional ex post alteration, in order to account for the coordination of short- and medium-term hydro resources. All such alterations may introduce significant deviation from optimality, measured in terms of social welfare, as discussed in Conejo et al.[2]. Although the introduction of coordination strategies in the auction model used by the PX is not a current practice, some sort of coordination must be performed ex post by the ISO, in order to comply with security issues.

When the day-ahead market takes the form of a power pool (PP), the market clearing procedure is performed by means of auction models which generally represent the generation and transmission systems accurately, including inter-temporal constraints, losses and congestion management. However, no sort of coordination strategy with the medium-term decisions is generally adopted in these models. In the PP the OM and the ISO are responsible for scheduling the system generation. Since the auction model used in the PP takes into account relevant constraints associated with generation and transmission systems, the dispatch calculated by this model does not need to be altered by ex post procedures in order to take into account congestion and losses in the transmission system. However, since a coordination strategy between short- and medium-terms is generally neglected in the auction models, an ex post alteration in the dispatch calculated may still be needed, in order to prevent hydro scarcity or losses. Therefore, incorporating short- and medium-term coordination strategies in the auction model would avoid these dispatch alterations, also avoiding sub-optimality measured in terms of social welfare and cross-subsidies.
The offers of hydro units tend to be smaller than those submitted by thermal units. Therefore, there would be a tendency of fully dispatching most of the hydro units every day. This daily pattern of full scheduling could lead to future hydro scarcity whenever future inflows are low. Therefore, we can anticipate that if no sort of coordination is performed in a hydro-dominated system, the ISO could face problems in the medium-term, concerning hydro resources.

\section{Coordination strategies}

In this section, we discuss two alternative strategies for coordinating decisions of an auction model with medium-term energy resources. As already discussed in Section "The need to coordinate", since short- and medium-term scheduling problems do not result from a formal decomposition of a larger optimization problem, we use the theory of decomposition only as an inspiration for handling with the coordination problem.

Let a global two-stage decision process be represented by problem (1), such that $y \in Y \subset \mathbb{R}^{n_{y}}$ represents the vector of short-term decisions and $x \in X \subset \mathbb{R}^{n_{x}}$ represents the vector of medium-term decisions:

$$
\begin{aligned}
& \min _{x, y} f_{a}(x)+f_{b}(y) \\
& \text { s.t : } g(x) \leqslant 0 \\
& h(x, y) \leqslant 0 \\
& \sum_{p \in P} y_{p, t}=x_{t}, \quad \forall t \in T \\
& x_{t}^{\min } \leqslant x_{t} \leqslant x_{t}^{\max }, \quad \forall t \in T \\
& x \in X, y \in Y,
\end{aligned}
$$

where $f_{a}(x): \mathbb{R}^{n_{x}} \rightarrow \mathbb{R}, f_{b}(y): \mathbb{R}^{n_{y}} \rightarrow \mathbb{R}, g(x): \mathbb{R}^{n_{x}} \rightarrow \mathbb{R}^{m}$ and $h(x, y)$ $\mathbb{R}^{n_{x} n_{y}} \rightarrow \mathbb{R}^{p}, P$ is the set of periods (hours) of the short-term problem and $T$ is the set of intervals (days) of the medium-term problem, $y_{p, t}$ is the short-term decision associated with period $p$ of interval $t$, and $x_{t}$ is the long-term decision associated with interval $t$. For easiness of explanation of the decomposition methods, we highlight in (1) the equality constraints that integrate short- and medium-term decisions, as well as minimum and maximum limits in the mediumterm decisions. Some coordination techniques have been discussed in the literature to decompose the two-stage process into independent short- and a medium-term problems, such as Benders' decomposition, Dantzig-Wolfe decomposition, Lagrangian relaxation, among others. These independent problems are generally coordinated by means of iterative information exchanging.

\section{Benders decomposition}

In Benders decomposition, problem (1) is rewritten in terms of $x$ variables only, as shown in (2), by means of function $\alpha(x)$, which is responsible for mapping the decisions of short-term problem into the global two-stage problem:

$$
\begin{aligned}
\min _{x} & f_{a}(x)+\alpha(x) \\
\text { s.t }: & g(x) \leqslant 0 \\
& x_{t}^{\min } \leqslant x_{t} \leqslant x_{t}^{\max }, \quad \forall t \in T \\
& x \in X .
\end{aligned}
$$

Problem (2) is known as the medium-term problem, and the value of $\alpha(x)$ is obtained from the solution of the short-term problem described in (3):

$$
\begin{aligned}
\min _{y} & f_{b}(y) \\
\text { s.t }: & h(x, y) \leqslant 0 \\
& \sum_{p \in P} y_{p, t}=x_{t}, \quad \forall t \in T
\end{aligned}
$$$$
y \in Y \text {. }
$$ 
The dual associated with shot-term problem (3) is given by (4):

$\sup _{\lambda \geqslant 0}\left[\inf _{y \in Y}\left(f_{b}(y)+\lambda^{T} h(x, y)+\sum_{t \in T} \lambda_{t}\left(\sum_{p \in P} y_{p, t}-x_{t}\right)\right)\right]$,

where $\lambda$ is the vector of dual variables associated with the inequality constraints in (3). Problem (4) may also be put in the equivalent form (5):

$\min \alpha(x)$

s.t:

$$
\alpha(x) \geqslant \inf _{y \in Y}\left(f_{b}(y)+\lambda^{T} h(x, y)+\sum_{t \in T} \lambda_{t}\left(\sum_{p \in P} y_{p, t}-x_{t}\right)\right), \quad \forall \lambda \geqslant 0 .
$$

If specific conditions hold that the duality gap is null (see [9]), the solution of the dual problem (5) matches that of the primal shortterm problem (3). Therefore, the function $\alpha(x)$ in (5) is the same described in (2). Finally, the global two-stage process can be rewritten in (6), in terms of $x$ only:

$$
\begin{aligned}
\min _{x} & f_{a}(x)+\alpha(x) \\
\text { s.t }: & g(x) \leqslant 0 \\
& \alpha(x) \geqslant \inf _{y \in Y}\left(f_{b}(y)+\lambda^{T} h(x, y)+\sum_{t \in T} \lambda_{t}\left(\sum_{p \in P} y_{p, t}-x_{t}\right)\right), \forall \lambda \geqslant 0 \\
& x \in X .
\end{aligned}
$$

The constraints associated with $\alpha(x)$ in (6) are known as Bender's optimality cuts. For generalized Benders decomposition [9] feasibility cuts may also be added to problem (6). The coordination process between short- and medium-term problems is illustrated in Fig. 1. Note that the medium-term problem passes primal information $(x)$ to short-term, which returns dual $(\lambda)$ and primal $(y)$ information.

\section{Dantzig-Wolfe decomposition}

Consider the two-stage process (1). Note that in the equality constraint in (1) $x$ is written in terms of $y$. We can eliminate the equality constraints in (1) by substituting $x$ in the remaining functions of the two-stage process. Therefore, this problem can be rewritten in terms of $y$ only, as described in (7):

$$
\begin{aligned}
\min _{y} & f_{a}(y)+f_{b}(y) \\
\text { s.t }: & g(y) \leqslant 0 \\
& \quad h(y) \leqslant 0 \\
x_{t}^{\min } & \leqslant \sum_{p \in P} y_{p, t} \leqslant x_{t}^{\max }, \quad \forall t \in T \\
y & \in Y .
\end{aligned}
$$

We take the partial Lagrangian dual of the problem (7) by dualising the constraints associated with minimum and maximum limits in $x$, as shown in (8):

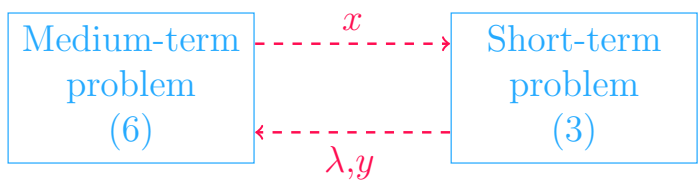

Fig. 1. Coordination process between short- and medium-term problems in Benders decomposition.

$$
\max _{\pi \geqslant 0, \eta \geqslant 0}\left\{\begin{array}{l}
\inf _{y}\left[f_{a}(y)+f_{b}(y)-\sum_{t \in T} \pi_{t}\left(\sum_{p \in P} y_{p, t}-x_{t}^{\max }\right)-\sum_{t \in K} \eta_{t}\left(x_{t}^{\min }-\sum_{p \in P} y_{p, t}\right)\right] \\
\text { s.t: } g(y) \leqslant 0 \\
h(y) \leqslant 0 \\
y \in Y .
\end{array}\right.
$$

Under specific convexity conditions [11], the solution of the dual problem (8) matches the solution of the primal problem (7). The dual problem can be rewritten as in (9):

$$
\max _{\pi \geqslant 0, \eta \geqslant 0}\left\{\begin{array}{l}
\sum_{t \in T} \pi_{t} x_{t}^{\max }-\sum_{t \in T} \eta_{t} x_{t}^{\min } \\
+\inf _{y}\left[f_{a}(y)+f_{b}(y)-\sum_{t \in T} \sum_{p \in P} \pi_{t} y_{p, t}+\sum_{t \in T} \sum_{p \in P} \eta_{t} y_{p, t}\right] \\
\text { s.t : } \quad g(y) \leqslant 0 \\
\quad h(y) \leqslant 0 \\
y \in Y .
\end{array}\right.
$$

We can separate (9) in two problems: the medium-term problem shown in (10) and the short-term problem shown in (11).

$$
\begin{aligned}
& \max _{\pi \geqslant 0, \eta \geqslant 0}\left\{\sum_{t \in T} \pi_{t} x_{t}^{\max }-\sum_{t \in T} \eta_{t} x_{t}^{\min }+v(\pi, \eta)\right. \\
& v(\pi, \eta)=\left\{\begin{array}{c}
\inf _{y}\left(f_{a}(y)+f_{b}(y)+\sum_{t \in T} \sum_{p \in P} \pi_{t} y_{p, t}-\sum_{t \in T} \sum_{p \in P} \eta_{t} y_{p, t}\right) \\
\text { s.t }: \begin{array}{l}
g(y) \leqslant 0 \\
h(y) \leqslant 0 \\
y \in Y .
\end{array}
\end{array}\right.
\end{aligned}
$$

This coordination process is illustrated in Fig. 2. The medium-term problem passes dual information $(\pi, \eta)$ to the short-term problem, which returns primal information $(y)$.

\section{Multi-period hydrothermal auction model with medium-term coordination}

We propose a Multi-period Hydrothermal Auction (MHA) model which involves a medium-term coordination. In Sections "Constants", "Variables" and "Sets", we describe constants, variables and sets related to the model formulation. In Section "Formulation", we detail the formulation of the objective function and constraints describing the MHA model. The following indexes are used: $p$ for periods, $k$ for nodes, $i$ for loads, $j$ for generating units, and $k l$ for lines connecting buses $k$ an $l$.

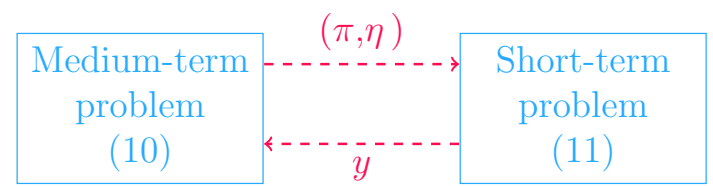

Fig. 2. Coordination process between short- and medium-term problems in Dantzig-Wolfe decomposition. 
Nomenclature

Constants

\begin{tabular}{|c|c|}
\hline$\alpha_{k l}^{b}$ & $\begin{array}{l}\text { slope of segment (block) } b \text { associated with the } \\
\text { piecewise linearization process of the power losses of } \\
\text { line } k l\end{array}$ \\
\hline$\lambda_{D_{i m}}^{p}$ & $\begin{array}{l}\text { price bid by the demand } i \text {, in period } p \text {, to buy power } \\
\text { block } m(\$ / \mathrm{MW} \text { h) }\end{array}$ \\
\hline$\lambda_{G_{j n}}^{p}$ & $\begin{array}{l}\text { price offered by the generator unit } j \text {, in period } p \text { to sell } \\
\text { power block } n(\$ / M W h)\end{array}$ \\
\hline$\Delta \delta$ & $\begin{array}{l}\text { upper bound on the blocks of the piecewise } \\
\text { linearization process of the power losses (rad) }\end{array}$ \\
\hline$B_{k l}$ & susceptance of line $k l(\mathrm{pu})$ \\
\hline$C_{j}^{0}$ & fixed cost of the generator unit $j(\$ / h)$ \\
\hline$C_{j}^{s d}$ & shut-down cost of the generator unit $j(\$)$ \\
\hline$C_{j}^{s u}$ & start-up cost of the generator unit $j(\$)$ \\
\hline$D P_{j}$ & minimum down time of unit $j(\mathrm{~h})$ \\
\hline$G_{k l}$ & conductance of line $k l(\mathrm{pu})$ \\
\hline$P_{j}^{\text {on }}$ & $\begin{array}{l}\text { number of periods unit } j \text { must be online at the } \\
\text { beginning of the market horizon due to its minimum up } \\
\text { time constraint (h) }\end{array}$ \\
\hline$P_{j}^{\text {off }}$ & $\begin{array}{l}\text { number of periods unit } j \text { must be offline at the } \\
\text { beginning of the market horizon due to its minimum } \\
\text { down time constraint }(h)\end{array}$ \\
\hline$N_{D_{i}}^{p}$ & $\begin{array}{l}\text { number of blocks of energy bid demanded by consumer } \\
i \text { in period } p\end{array}$ \\
\hline$N_{G_{j}}^{p}$ & $\begin{array}{l}\text { number of blocks of energy bid offered by unit } j \text { in } \\
\text { period } p\end{array}$ \\
\hline$N P$ & number of periods of the market horizon \\
\hline$P_{k l}^{\max }$ & maximum capacity of the line $k l(\mathrm{MW})$ \\
\hline$P_{D_{i m}}^{\max }$ & $\begin{array}{l}\text { upper limit of the power block } m \text { demanded by } \\
\text { consumer } i(\mathrm{MW})\end{array}$ \\
\hline$P_{G_{j}}^{\max }$ & $\begin{array}{l}\text { upper limit for the power output of generator unit } j \\
\text { (MW) }\end{array}$ \\
\hline$P_{G_{j n}}^{\max }$ & $\begin{array}{l}\text { upper limit of the power block } n \text { offered by generator } \\
\text { unit } j \text { in period } t(\mathrm{MW})\end{array}$ \\
\hline$S_{j}^{0}$ & $\begin{array}{l}\text { time periods unit } j \text { has been offline at the beginning of } \\
\text { the market horizon }(h)\end{array}$ \\
\hline$S D_{j}$ & shut-down ramp limit of unit $j(\mathrm{MW} / \mathrm{h})$ \\
\hline$S U_{j}$ & start-up ramp limit of unit $j(\mathrm{MW} / \mathrm{h})$ \\
\hline$R D_{j}$ & ramp-down limit of unit $j(\mathrm{MW} / \mathrm{h})$ \\
\hline$R U_{j}$ & ramp-up limit of unit $j(\mathrm{MW} / \mathrm{h})$ \\
\hline$U_{j}^{0}$ & $\begin{array}{l}\text { time periods unit } j \text { has been online at the beginning of } \\
\text { the market horizon }(\mathrm{h})\end{array}$ \\
\hline$U P_{j}$ & minimum up time of unit $j(\mathrm{~h})$ \\
\hline$V_{j}^{0}$ & $\begin{array}{l}\text { initial commitment status of unit } j \text { ( } 1 \text { if it is online, } 0 \\
\text { otherwise) }\end{array}$ \\
\hline
\end{tabular}

\section{Variables}

\footnotetext{
$\delta_{k l}^{p} \quad$ variable used in phe linearization process of the power losses, for representing the absolute value of the angular difference between nodes $k$ and $l$ in the period $p(\mathrm{rad})$

$\theta_{k}^{p} \quad$ voltage angle at node $k$ in hour $p$ (rad)

$\theta_{k l}^{p} \quad$ difference in voltage angles in nodes $k$ and $l$ in period $p$, given by $\theta_{k l}^{p}=\theta_{k}^{p}-\theta_{l}^{p}$
}

total load located at node $k$ in period $p(\mathrm{MW} / \mathrm{h})$

$g_{k}^{p} \quad$ total power generation at node $k$ in period $p(\mathrm{MW} / \mathrm{h})$

$p_{D_{i}}^{p} \quad$ power demanded by consumer $i$ in period $p$ (MW)

$p_{G_{j}}^{p} \quad$ power output of unit $j$ in period $p$ (MW)

$p_{D_{\text {im }}}^{p} \quad$ power associated with block $m$ consumed by load $i$ in period $p(\mathrm{MW})$

$p_{G_{j n}}^{p} \quad$ power output associated with block $n$ generated by unit $j$ in period $p(\mathrm{MW})$

$\bar{p}_{j}^{p} \quad$ maximum available power output of unit $j$ in period

\section{$p(\mathrm{MW})$}

$p_{k l}^{p}\left(\theta_{k l}^{p}\right) \quad$ power flow in line $(k, l)$ at node $k$ in period $p$ (MW)

$v_{j}^{p} \quad$ binary variable which is equal to 1 if unit $j$ is online in period $p$, or 0 otherwise

$y_{j}^{p} \quad$ binary variable which is equal to 1 if unit $j$ is started-

$y_{j} \quad$ up at the beginning of period $p$, or 0 otherwise

$z_{j}^{p} \quad$ binary variable which is equal to 1 if unit $j$ is shut-

down at the beginning of period $p$, or 0 otherwise

$\mathcal{L}_{k l}\left(\theta_{k l}^{p}\right)$ power losses in line $(k, l)$ at node $k$ in period $p(\mathrm{MW})$

Sets

$B$ the set of blocks for the linearization process of the power losses

$H$ set of indexes of hydro generating units

I set of indexes of periods of the demands

$J$ set of indexes of generating units

$K \quad$ set of indexes of network buses

$P \quad$ set of indexes of periods of the market horizon

$\Omega_{k}^{p} \quad$ set of lines directly linked to bus $k$ in period $p$

$\Lambda_{k}^{p} \quad$ set of generating units located at bus $k$ in period $p$

$\Psi_{k}^{p} \quad$ set of demands located at bus $k$ in period $p$

\section{Formulation}

The MHA model for a pool-based electricity market, is concerned with the maximization of the declared social welfare while enforcing the network constraints, minimum up and down time limits, ramp rate limits and a coordination scheme between short- and medium-term scheduling for hydroelectric units.

\section{Objective function}

The goal of the Market Operator (MO) is to maximize the net social welfare. For a single period, the social welfare is defined as the sum of the consumer surplus and the producer surplus in that period. For a multi-period auction, the net social welfare is defined as the sum of the social welfare functions over all the periods. Additional offer terms for generating units may be included to consider the start-up, shut-down and no-load costs. Thus, the objective function to be maximized can be expressed by (12):

$$
\begin{aligned}
\mathrm{OF}\left(p_{D_{\text {im }}}^{p}, p_{G_{j n}}^{p}, v_{j}^{p}, y_{j}^{p}, z_{j}^{p}\right)= & \sum_{p \in P} \sum_{i \in I} \sum_{m=1}^{N_{D i}^{p}} \lambda_{D_{i m}}^{p} p_{D_{i m}}^{p} \\
& -\sum_{p \in P} \sum_{j \in J}\left(v_{j}^{p} C_{j}^{0}+y_{j}^{p} C_{j}^{s u}+z_{j}^{p} C_{j}^{s d}+\sum_{n=1}^{N_{G j}^{p}} \lambda_{G_{j n}}^{p} p_{G_{j n}}^{p}\right) .
\end{aligned}
$$


In Eq. (12), the first term is related to the buying energy bids whereas the second term includes generation offers associated with no-load costs, start-up, shut-down costs and of the energy selling, respectively.

\section{Network-constraints}

The following expressions represent the set of networkconstraints related to every node $k$ and every line $k l$ of the system over the market horizon $P$.

$g_{k}^{p}-d_{k}^{p}=\sum_{l \in \Omega_{k}^{p}} p_{k l}\left(\theta_{k l}^{p}\right), \quad \forall k \in K, \forall p \in P$,

$p_{k l}\left(\theta_{k l}^{p}\right)=B_{k l} p_{k l}^{p}+\frac{\mathcal{L}_{k l}\left(\theta_{k l}^{p}\right)}{2}, \quad \forall k \in K, \forall l \in \Omega_{k}^{p}, p \in P$,

$-P_{k l}^{\max } \leqslant p_{k l}\left(\theta_{k l}^{p}\right) \leqslant P_{k l}^{\max }, \quad \forall k \in K, \forall l \in \Omega_{k}^{p}, p \in P$,

where

$g_{k}^{p}=\sum_{j \in \Lambda_{k}^{p}} p_{G_{j}}^{p}, \quad \forall k \in K, \forall p \in P$,

$p_{G_{j}}^{p}=\sum_{n=1}^{N_{G_{j}}^{p}} p_{G_{j n}}^{p}, \quad \forall j \in J, \forall p \in P$,

$d_{k}^{p}=\sum_{i \in \Psi_{k}^{p}} p_{D_{i}}^{p}, \quad \forall k \in K, \forall p \in P$

$p_{D_{i}}^{p}=\sum_{m=1}^{N_{D_{i}}^{p}} p_{D_{\text {im }}}^{p}, \quad \forall i \in I, \forall p \in P$,

$\mathcal{L}_{k l}\left(\theta_{k l}^{p}\right)=G_{k l}\left(\theta_{k l}^{p}\right)^{2}, \quad \forall k \in K, \forall l \in \Omega_{k}^{p}, p \in P$.

The constraints (13) enforce the power balance at every node $k$ and every period $p$. The constraints (14) represent the active power flows in the transmission lines. The constraints (15) enforce flow limits in line $k l$ at every hour $p$.

The representation of power losses through Eq. (20) would lead to a nonlinear MHA model. In order to formulate the auction model as a mixed-integer linear programming problem, which is considered a more transparent procedure, some techniques have been proposed to linearize power losses $(20)[15,16]$. In this paper we adopted the technique proposed in Motto et al. [15], which consists of a linear piecewise approximation of (20), obtained by using $B$ linear blocks as shown in Fig. 3. In order to consider the linearization in the positive orthant only, Motto et al. [15] introduce the definition of the absolute value of angle difference between nodes $k$ and $l$, as shown in (21):

$\delta_{k l}^{p}=\left|\theta_{k l}^{p}\right|, \quad \forall k \in K, \forall l \in \Omega_{k}^{p}, \forall p \in P$.

By using definition (21) and from Fig. 3, the authors also derive the value of $\delta_{k l}^{p}$ as being equal to the sum of values $\delta_{k l}^{b, p}$ in each block $b$ of the linearization process, as shown in (22):

$\delta_{k l}^{p}=\sum_{b=1}^{B} \delta_{k l}^{b, p}, \quad \forall k \in K, \forall l \in \Omega_{k}^{p}, \forall p \in P$.

Therefore, the power losses $\mathcal{L}_{k l}\left(\theta_{k l}^{p}\right)$ in each line $k l$ are formulated as in (23):

$\mathcal{L}_{k l}\left(\theta_{k l}^{p}\right)=G_{k l} \sum_{b=1}^{B} \alpha_{k l}^{b} \delta_{k l}^{b, p}, \quad \forall k \in K, \forall l \in \Omega_{k}^{p}, \forall p \in P$,

where

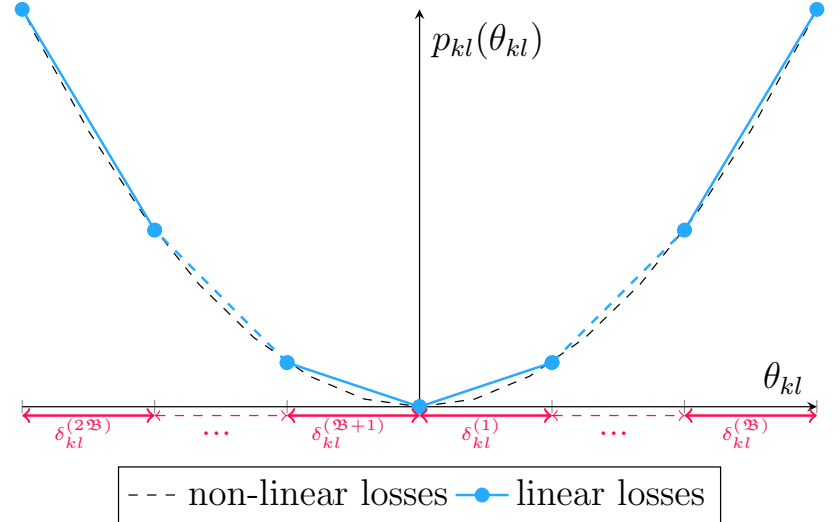

Fig. 3. Piecewise linear approximation for the loss function. Source: Motto et al. [15].

$0 \leqslant \delta_{k l}^{b, p} \leqslant \Delta \delta, \quad \forall k \in K, \forall l \in \Omega_{k}^{p}, \forall b \in B, \forall p \in P$

$\alpha_{k l}^{b}=(2 b-1) \Delta \delta, \quad \forall k \in K, \forall l \in \Omega_{k}^{p}, \forall b \in B$.

The expression for $\alpha_{k l}^{b}$ given in (25) can also be derived from Fig. 3. To avoid the non-differentiable modular function in (21) the authors also use a linear representation of this function, by replacing (21) by the sets of constraints (26)-(28):

$\delta_{k l}^{p}=\delta_{k l}^{p^{+}}+\delta_{k l}^{p^{-}}, \quad \forall k \in K, \forall l \in \Omega_{k}^{p}, \forall p \in P$,

$\theta_{k l}^{p}=\delta_{k l}^{p^{+}}-\delta_{k l}^{p^{-}}, \quad \forall k \in K, \forall l \in \Omega_{k}^{p}, \forall p \in P$

$\delta_{k l}^{p^{+}} \geqslant 0, \quad \delta_{k l}^{p^{-}} \geqslant 0, \quad \forall k \in K, \forall l \in \Omega_{k}^{p}, \forall p \in P$.

In summary, the nonlinear power losses given in (20) are replaced by the linearized losses in (23) and the set of constraints (24)(28) is introduced in the MHA model.

\section{Thermal constraints}

The following expressions represent the set of constraints related to every generating unit $j$ and every consumer $i$ over the market horizon $P$. The set of thermal constraints used in the MHA model is strongly based on the auction model described by Arroyo and Conejo [1], which provide a precise representation for such constraints. Whenever possible, the notation used to represent the thermal constraints in Arroyo and Conejo [1] was maintained in this paper.

Power output limits. The formulation of the power output limits is as follows:

$P_{G_{j 1}}^{\max ^{p}} v_{j}^{p} \leqslant p_{G_{j}}^{p} \leqslant \bar{p}_{j}^{p}, \forall j \in J, \forall p \in P$,

$p_{G j n}^{p} \leqslant P_{G j n}^{\max ^{p}}, \forall j \in J, \forall p \in P, \forall n=1, \ldots, N_{G j}^{p}$.

Constraints (29) set the generating limits of each unit $j$ for each period $p$. The power output is enforced to be smaller than the maximum available power output, $\bar{p}_{j}^{p}$, which expresses the real availability of power by taking into consideration the effect of all ramp rate limits over the power availability (as described in the next section). Constraints (29) also set the minimum power generated by a unit $j$ in a period $p$ as the upper limit of the first power block offered by unit $j$ in period $p$. Constraints (30) sets upper limits for the power output associated with each offer block provided by each generating unit in each period. 
Maximum available power output and ramp rate limits. Maximum available power output and ramp rate limit constraints are formulated as follows:

$\bar{p}_{j}^{p} \leqslant P_{G_{j}}^{\max ^{p}}\left[v_{j}^{p}-z_{j}^{p+1}\right]+z_{j}^{p+1} S D_{j}, \quad \forall j \in J, \forall p \in P$,

$\bar{p}_{j}^{p} \leqslant p_{G_{j}}^{p-1}+R U_{j} v_{j}^{p-1}+S U_{j} y_{j}^{p}, \quad \forall j \in J, \forall p \in P$,

$p_{G_{j}}^{p-1}-p_{G_{j}}^{p} \leqslant R D_{j} v_{j}^{p}+S D_{j} z_{j}^{p}, \quad \forall j \in J, \forall p \in P$,

where

$P_{G_{j}}^{\max ^{p}}=\sum_{n=1}^{N_{G_{j}}^{p}} P_{G_{j n}}^{\max ^{p}}, \quad \forall j \in J, \forall p \in P$.

Constraints (31) and (32) set the upper limits of the maximum available power output by taking into account the unit actual capacity, start-up, shut-down and ramp rate limits. The set of constraints (33) imposes the ramp-down rate limits as well as the shutdown ramp rate limits.

Minimum up time. Linear expressions of minimum up time constraints are presented as follow:

$\sum_{p=1}^{P_{j}^{\text {on }}}\left[1-v_{j}^{p}\right]=0, \quad \forall j \in J$,

$\sum_{w=p}^{p+U P_{j}-1} v_{j}^{w} \geqslant U P_{j} y_{j}^{p}, \quad \forall j \in J, \forall p=P_{j}^{\text {on }}+1, \ldots, N P-U P_{j}+1$

$\sum_{w=p}^{N P}\left[v_{j}^{w}-y_{j}^{p}\right] \geqslant 0, \quad \forall j \in J, \forall p=N P-U P_{j}+2, \ldots, N P$,

where

$P_{j}^{\text {on }}=\operatorname{Min}\left\{N P,\left[U P_{j}-U_{j}^{0}\right] V_{j}^{0}\right\}, \quad \forall j \in J$.

Constraints (34) are related to the initial statuses of the units. $P_{j}^{\text {on }}$ is the number of initial periods during which unit $j$ must be online. Constraints (35) are used for periods following $P_{j}^{\text {on }}$, and ensure the enforcement of minimum up time during all the possible sets of consecutive periods of size $U P_{j}$. Finally, constraints (36) are needed for the last $U P_{j}-1$ periods.

Minimum down time. Linear expressions for minimum down time constraints, expressing the number of periods during which unit $j$ must be off-line, are presented below, and are analogous to (34)-(36):

$\sum_{p=1}^{P_{j}^{\text {off }}} v_{i}^{p}=0, \quad \forall j \in J$

$\sum_{w=p}^{p+D P_{j}-1}\left[1-v_{j}^{w}\right] \geqslant D P_{j} z_{j}^{p}, \quad \forall j \in J, \forall p=P_{j}^{\mathrm{off}}+1, \ldots, N P-D P_{j}+1$,

$\sum_{w=p}^{N P}\left[1-v_{j}^{w}-z_{j}^{p}\right] \geqslant 0, \quad \forall j \in J, \forall p=N P-D P_{j}+2, \ldots, N P$,

where

$P_{j}^{\text {off }}=\operatorname{Min}\left\{N P,\left[D P_{j}-S_{j}^{0}\right]\left[1-V_{j}^{0}\right]\right\}, \quad \forall j \in J$.
Logical status of commitment.

$y_{j}^{p}-z_{j}^{p}=v_{j}^{p}-v_{j}^{p-1}, \quad \forall j \in J, \forall p \in P$,

$y_{j}^{p}+z_{j}^{p} \leqslant 1, \quad \forall j \in J, \forall p \in P$.

Constraints (40) and (41) establish logical values for the binary variables, avoiding simultaneous commitment and decommitment of units.

Upper limit power load

Eq. (42) set the upper limit of the hourly power consumed $p_{D_{i m}}^{p}$ in each block $m$ by each consumer $i$.

$p_{D_{\text {im }}}^{p} \leqslant P_{D_{\text {im }}}^{\max ^{p}}, \quad \forall i \in I, \forall m \in N_{D i}^{p}, \forall p \in P$.

Auction model with primal coordination strategy

In this section we introduce, in the auction model, the primal coordination strategy inspired by Benders decomposition described in Section "Benders decomposition". In Benders decomposition the short-term model is altered by the introduction of additional constraints as shown in Fig. 1. For the auction model here proposed these constraints can be written as in (43) for each hydro unit:

$\sum_{p \in P} p_{G_{j}}^{p}=M_{j}, \quad \forall j \in H$,

where $M_{j}$ is the daily energy target previously calculated by the medium-term generation model. The model resulting from such coordination approach is a network-constrained multi-period hydrothermal auction model with primal coordination strategy, simply refered to as $\left(\mathrm{MHA}_{P}\right)$, which is shown in (44):

$\operatorname{MHA}_{P}\left\{\begin{array}{ll}\max & (12) \\ \text { s.t : } & (13)-(19) \\ & (23)-(42) \\ & \sum_{p \in P} p_{G_{j}}^{p}=M_{j}, \quad \forall j \in H\end{array}\right.$.

\section{Auction model with dual coordination strategy}

The auction model with dual coordination strategy is inspired in the Dantzig-Wolfe decomposition described in Section "DantzigWolfe decomposition". In the Dantzig-Wolfe decomposition the short-term model is altered by the introduction of additional terms in the objective function. Therefore, the auction model here proposed can be written as in (45):

$\mathrm{MHA}_{D}\left\{\begin{array}{ll}\max & (12)+\sum_{j \in H} \pi_{j} p_{G_{j}}^{p}+\sum_{j \in H} \eta_{j} p_{G_{j}}^{p} \\ \text { s.t : } & (13)-(19) \\ & (23)-(42)\end{array}\right.$,

where $\pi_{j}$ and $\eta_{j}$ are the dual information associated with unit $j$, whose values are obtained from the Lagrange multipliers associated with the constraints representing maximum and minimum generation limits, respectively, in the medium-term model, as shown in Section "Dantzig-Wolfe decomposition".

\section{Auction model with primal and dual coordination strategy}

In the primal and dual coordination strategy we introduce both primal and dual information in the auction model. In this case, the 
energy targets are relaxed to a previously fixed range $\left[M_{j}^{\min }, M_{j}^{\max }\right]$. The resulting auction model is shown in (46):

$$
\mathrm{MHA}_{P D}\left\{\begin{array}{ll}
\max & (12)+\sum_{j \in H} \pi_{j} p_{G_{j}}^{p}+\sum_{j \in H} \eta_{j} p_{G_{j}}^{p} \\
\text { s.t : } \quad & (13)-(19) \\
& (23)-(42) \\
& M_{j}^{\min } \leqslant \sum_{p \in P} p_{G_{j}}^{p} \leqslant M_{j}^{\max }, \quad \forall j \in H .
\end{array} .\right.
$$

\section{Numerical results}

In this section we present the numerical results obtained with the proposed network-constrained MHA model described in Section "Multi-period hydrothermal auction model with mediumterm coordination". The main focus here is to evaluate the impact of the coordination strategies described in Section "Coordination strategies in hydrothermal auction models" in terms of nodal prices and the hourly hydrothermal dispatches calculated by the MHA model. For such a purpose, initially we solve a simplified version of the MHA model (base case), which totally neglects mediumterm coordination. Then, we solve three network-constrained MHA models using primal, dual and primal/dual coordination strategies, given by $\mathrm{MHA}_{P}, \mathrm{MHA}_{D}$ and $\mathrm{MHA}_{P D}$ respectively. Comparisons of such coordination strategies are then performed.

Numerical results were carried out with the IEEE RTS-1996 24-bus test system, whose data are detailed in Crespo et al. [4], Garcia-Bertrand et al. [8] and Grigg et al. [10]. We adapted the data for this system in order to represent a stronger hydro participation in the total generation. For such a purpose, thermal units 1, 2, 5 and $6(20 \mathrm{MW})$ and also units 3, 4, 7 and 8 (76 MW) were replaced by hydro generating units, all having generation capacity of $50 \mathrm{MW}$. Furthermore, thermal units 22 and 23 (400 MW) were also replaced by hydro units with $400 \mathrm{MW}$. Thermal generation is provided by units 9-11 (100 MW), 12-14 (197 MW), 15-19 (12 MW), $20,21,30,31(350 \mathrm{MW})$ and 32 (350 MW). Therefore, the total hydro generation capacity is $1200 \mathrm{MW}$, while the total thermal capacity is $1921 \mathrm{MW}$.

Also, we reduced the capacity of the transmission lines 14-16 and $16-17$ from $500 \mathrm{MW}$ to $200 \mathrm{MW}$ and $300 \mathrm{MW}$, respectively, in order to artificially induce congestion in the transmission network. We adopted null values for no-load generation costs as well as for shut-down and start-up costs. The offer blocks of prices for all hydro units were $\$ 4.96, \$ 5.12, \$ 5.34$ and $\$ 5.63$, respectively for blocks 1-4. For simplicity, the same offer block structure was adopted for all 24 periods of the auction model.

In order to artificially generate price spikes, we increased the bids of consumers $9-11$ and $14-15$ in $10 \%$, and the bids prices of consumers $6-9,16-20$ and $22-23$ in $24 \%$, while the bid price of consumer 5 was reduced in $5 \%$. The upper limits of bid blocks for consumers were also changed. We reduced the upper limits of bid blocks for consumers 2 and 3 in 10\% and 15\%, respectively; the upper limits of bid blocks for consumers 5, 10-17 and 22-24 were reduced in 20\%; finally, we increased the upper limits of bid blocks for consumers 7, 9, 18, 19 in $10 \%, 20 \%, 40 \%$ and $50 \%$, respectively. The remaining block data were kept as described in Crespo et al. [4], Garcia-Bertrand et al. [8] and Grigg et al. [10].

We solved all the MHA formulations for the test system using IBM ILOG CPLEX Optimization Studio 12.6. The total hourly generation dispatch and the transmission losses obtained for the base case are shown in Fig. 4. Note that the highest levels of consumption occur in periods 8,18 and 19 , while the lowest levels occur in periods $3-5$.

For solving $\mathrm{MHA}_{P}$ we need to adopt specific daily energy targets for each hydro unit. In practical applications, these energy targets are obtained by solving medium-term equilibrium models. Here, we adopted the following energy targets: $10.8 \mathrm{MW}$ for hydro units $1-8,48 \mathrm{MW}$ for hydro units 22 and $23,6 \mathrm{MW}$ for hydro units 25-29. These energy targets correspond to values of respectively $50 \%, 90 \%$ and $50 \%$ of the total daily generation calculated in the base case for such units. For solving $\mathrm{MHA}_{D}$ we adopted the following penalty terms: $20.85 \$ / M W$ for units $1-4,20.90 \$ / M W$ for units 5-8, 5.31 and 5.24 \$/MW for units 22 and 23, respectively, and $5.26 \$ / \mathrm{MW}$ for units $24-29$. For solving $\mathrm{MHA}_{P D}$ we adopted the same penalty terms used in the dual coordination for problem $\mathrm{MHA}_{D}$ and the following ranges for the energy targets: 10.8-12 (MW) for units 1-8, 38.5-57.6(MW) for units 22-23, and 4.8-7.2 (MW) for units 24-29.

The models $\mathrm{MHA}_{P}, \mathrm{MHA}_{D}$ and $M H A_{P D}$, involving different coordination strategies were solved for the test system. Total hydro and thermal generation for each period and each model is shown in Fig. 5. In this figure, we observe that the solution provided by the base case have higher hydro participation than those obtained by the models having some sort of coordination strategy. This is an expected result since the offer costs of hydro units are lower than those of thermal units; therefore, if no coordination with mediumterm planning is enforced, the system will tend to fully dispatch the hydro units in all periods. When the coordination strategy is introduced, the models tend to reduce total hydro generation in order to enforce medium-term reliability constraints, which are related to the rational use of water resources. This result also emphasizes that a reliable and secure operation of a hydrothermal system tends to save more energy (water) in the hydro plants by increasing short-term thermal participation in the total generation. Since thermal generation is generally more expensive than hydro one, a reliable and secure operation tends to be more expensive.

By analyzing the total hydro and thermal generation calculated by the coordination strategies in Fig. 5 , we observe that primal coordination used in $\mathrm{MHA}_{p}$ model calculates the highest thermal participation in all periods, while the dual coordination strategy used in $\mathrm{MHA}_{D}$ calculates the lowest ones. We note that since primal coordination imposes hard equality constraints represented by the daily hydro generation targets, these constraints may be too rigid, in the sense that the amounts of daily energy must be strictly enforced. On the other hand, the dual coordination in $\mathrm{MHA}_{D}$ model tends to be more flexible in terms of enforcing security and reliability in the medium-term, since less energy (water) is stored in the reservoirs in the short-term. The dispatch obtained by the primal-dual coordination strategy is a intermediate solution.

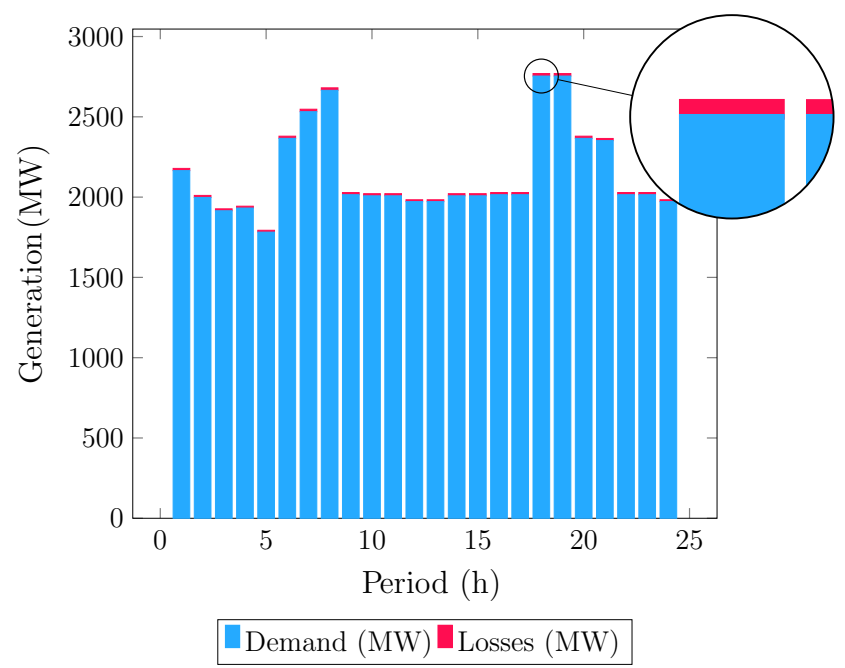

Fig. 4. Total hourly generation dispatch obtained for the base case. 


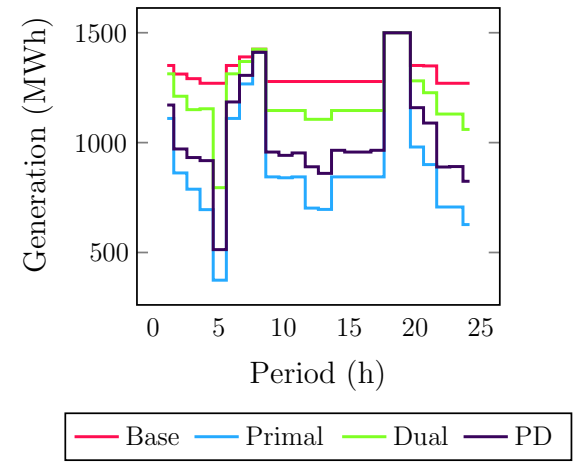

(a) Total hydro Generation.

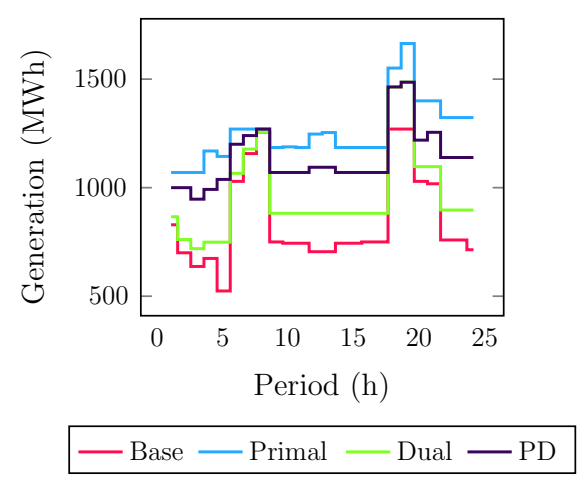

(b) Total thermal generation.

Fig. 5. Total hydro and thermal generation for each period.

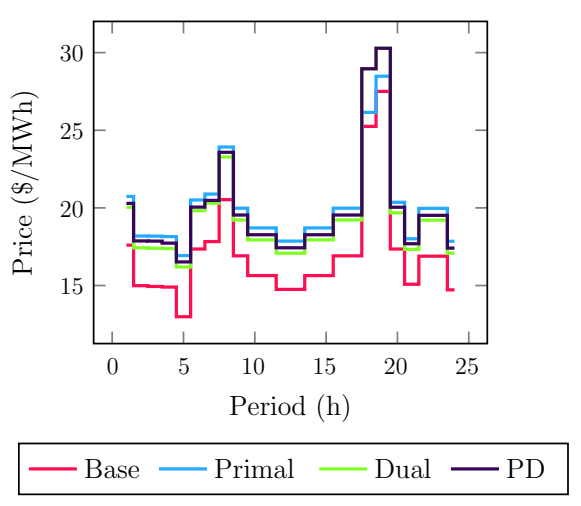

(a) Medium nodal prices for each period (price evolution in time).

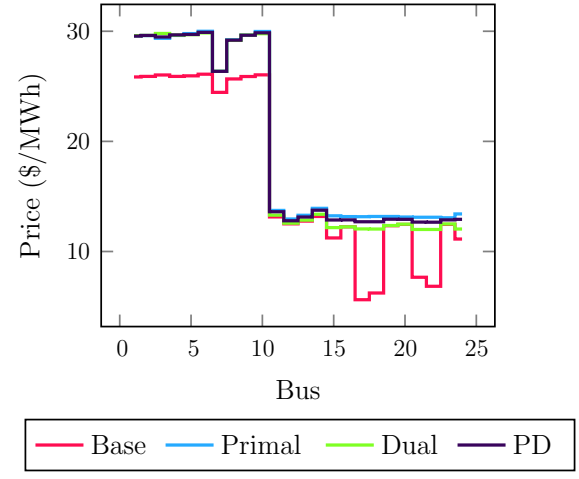

(b) Medium daily prices for each bus (spatial distribution of prices).

Fig. 6. Medium nodal prices for each period and medium daily prices for each bus.

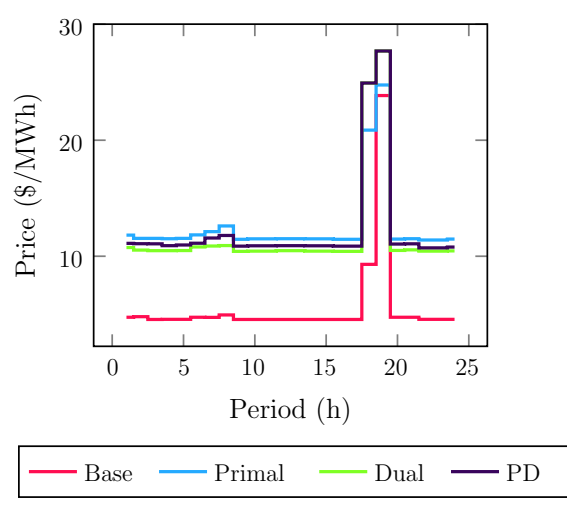

(a) Minimum nodal prices.

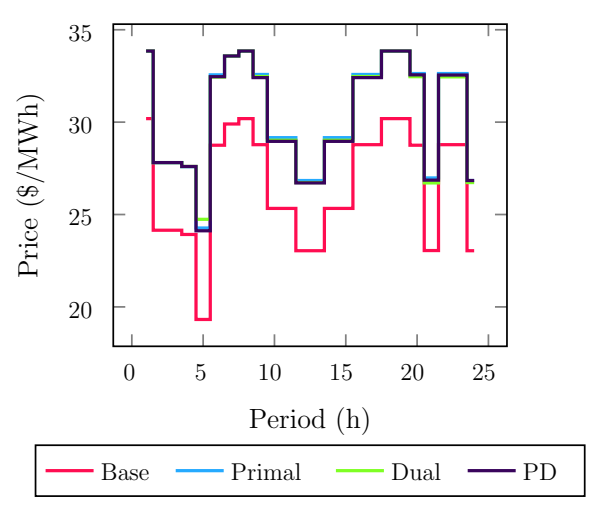

(b) Maximum nodal prices.

Fig. 7. Minimum and maximum nodal prices in the transmission network for each period.

This strategy is not excessively rigid in its coordination (such as in the $\mathrm{MHA}_{P}$ model), which strictly enforces the energy targets and stores a conservative amount of energy in the reservoirs (more reliable and secure), nor excessively flexible (such as in the MHA ${ }_{D}$ ), which uses a higher amount of water in the short-term, leaving the reservoirs with less amount of stored energy (less reliable and insecure). The energy prices obtained by all strategies are also analyzed in this section. We emphasize that since the transmission system is represented in detail, and since a coordination procedure is embedded in the MHA auction models, the prices calculated by such models implicitly represent the costs associated with transmission losses and congestion as well as the costs associated with medium-term coordination. This implies that no additional ex post alteration in the energy dispatch and prices are necessary in order to take these operational constraints into account. However, since hydro constraints, are neglected in the MHA model, small 
additional ex post corrections in energy dispatch and prices may be necessary.

We note that the energy prices vary both for each period (time variation) as well as for each transmission bus (spatial variation). Therefore, in order to compare the prices calculate by the models, we calculate the medium price for each period $t$, denoted by $\hat{\lambda}^{t}$, according to Eq. (47) and the medium daily price for each bus $k$, denoted by $\hat{\lambda}_{k}$, according to Eq. (48):

$\hat{\lambda}^{t}=\sum_{k=1}^{N B} \frac{\lambda_{k}^{t}}{N B}, \quad \forall t=1, \ldots, T$,

$\hat{\lambda}_{k}=\sum_{t=1}^{T} \frac{\lambda_{k}^{t}}{N T}, \quad \forall k=1, \ldots, N B$,

where $N B$ is the number of buses. Note that $\hat{\lambda}^{t}$ allows for the analysis of time evolution of prices, while $\hat{\lambda}_{k}$ allows for the analysis of the spatial distribution of prices in the transmission system. These medium price values are shown in Fig. 6 for the base case and also for $\mathrm{MHA}_{P}, \mathrm{MHA}_{D}$ and $\mathrm{MHA}_{P D}$ models. From Fig. 6(a), we note that the profile of medium nodal prices $\hat{\lambda}^{t}$ tends to follow the generation-consumption profile shown in Fig. 4 for all cases, as expected. By analyzing the spatial distribution of prices $\hat{\lambda}_{k}$ depicted in Fig. 6(b), we observe that the congestions in lines 14-16 and 16-17 breaks the transmission system into two subregions with similar medium daily prices. Minimum and maximum prices are illustrated in Fig. 7. We observe that a secure and reliable operation has also a higher market clearing price in all periods.

\section{Conclusions}

This paper investigates the coordination of decisions calculated by a network-constrained multi-period auction model of hydrothermal systems with the decisions calculated by a medium-term model. The need to introduce such coordination strategies is discussed from the perspective of a market operator seeking for the optimization of the social welfare but also the optimization of system security and reliability. Three alternative strategies for coordination are investigated: the first one incorporates additional constraints in the auction model associated with primal information provided by the medium-term model; the second one uses dual information concerning the value of the medium-term generation and introduces penalty functions in the objective function of the auction model; the third approach introduces primal and dual information of the medium-term model in the auction model. These strategies are compared by means of simulation results involving the 24-bus IEEE reliability test-system. The first strategy presents the best solution in terms of security and reliability since the smallest share of hydro generation is dispatched, but also the most expensive one, due to the high share of thermal generation. The second strategy dispatches the highest share of hydro generation, presenting the less secure but also the less expensive coordinating strategy. The third approach presented results that can be viewed as a good trade-off between security and costs. The results show that the coordination strategies have significant impact on market clearing prices and generation scheduling, since coordination tends to reduce hydro participation in the total generation in order to enforce reliability and security constraints.

\section{Acknowledgments}

This work was financed by Coordenação de Aperfeiçoamento de Pessoal de Nível Superior CAPES, by Conselho Nacional de Desenvolvimento Científico e Tecnológico CNPq, and also by Fundação de Amparo à Pesquisa Científica do Estado de São Paulo FAPESP, Brazil.

\section{References}

[1] Arroyo J, Conejo A. Multiperiod auction for a pool-based electricity market. IEEE Trans Power Syst 2002;17(4):1225-31.

[2] Conejo A, Galiana F, Arroyo J, Garcia-Bertrand R, Chua CW, Huneault M. Economic inefficiencies and cross-subsidies in an auction-based electricity pool. IEEE Trans Power Syst 2003;18(1):221-8.

[3] Conejo AJ, Carrin M, Morales JM. Decision making under uncertainty in electricity markets. International series in operations research \& management science, vol. 153. Boston, MA: Springer US; 2010.

[4] Crespo JM, Usaola J, Fernandez JL. Optimal security-constrained power scheduling by Benders decomposition. Electric Power Syst Res 2007 (77):739-53.

[5] Forsund FR. Hydropower economics. 2007 ed. New York: Springer; 2007.

[6] Fosso O, Gjelsvik A, Haugstad A, Mo B, Wangensteen I. Generation scheduling in a deregulated system. The Norwegian case. IEEE Trans Power Syst 1999;14 (1):75-81.

[7] Franco P, Carvalho M, Soares S. A network flow model for short-term hydrodominated hydrothermal scheduling problems. IEEE Trans Power Syst 1994;9 (2):1016-22.

[8] Garcia-Bertrand R, Conejo AJ, Gabriel S. Electricity market near-equilibrium under locational marginal pricing and minimum profit conditions. Eur J Oper Res 2006(174):457-79.

[9] Geoffrion AM. Generalized Benders decomposition. J Optim Theory Appl $1972 ; 10(4): 237-60$.

[10] Grigg C, Wong P, Albrecht P, Allan R, Bhavaraju M, Billinton R, et al. The IEEE reliability test system-1996. IEEE Trans Power Syst 1999(3):1010-20.

[11] Grothey A. Decomposition methods for nonlinear nonconvex optimization problems. Ph.D thesis. Department of Mathematics, The University of Edinburgh, Edinburgh; 2001.

[12] Kardakos E, Simoglou C, Bakirtzis A. Short-term electricity market simulation for pool-based multi-period auctions. IEEE Trans Power Syst 2013;28 (3):2526-35.

[13] Li C, Johnson R, Svoboda A. A new unit commitment method. IEEE Trans Power Syst 1997;12(1):113-9.

[14] Li T, Shahidehpour M. Price-based unit commitment, a case of Lagrangian relaxation versus mixed integer programming. IEEE Trans Power Syst 2005;20 (4):2015-25.

[15] Motto A, Galiana F, Conejo A, Arroyo J. Network-constrained multiperiod auction for a pool-based electricity market. IEEE Trans Power Syst 2002;17 (3):646-53.

[16] Norbiato dos Santos T, Diniz A. A dynamic piecewise linear model for DC transmission losses in optimal scheduling problems. IEEE Trans Power Syst 2011;26(2):508-19.

[17] Oliveira ARL, Soares S, Nepomuceno L. Short term hydroelectric scheduling combining network flow and interior point approaches. Int J Electr Power Energy Syst 2005;27(2):91-9.

[18] Padhy N. Unit commitment-a bibliographical survey. IEEE Trans Power Syst 2004;19(2):1196-205.

[19] Pereira M, Pinto L. A decomposition approach to the economic dispatch of hydrothermal systems. IEEE Trans Power Ap Syst 1982;PAS-101(10):3851-60.

[20] Pereira M, Pinto L. Application of decomposition techniques to the mid-and short-term scheduling of hydrothermal systems. IEEE Trans Power Ap Syst 1983:PAS-102(11):3611-8.

[21] Renaud A. Daily generation management at Electricite de France: from planning towards real time. IEEE Trans Autom Control 1993;38(7):1080-93.

[22] Reneses J, Centeno E, Barquin J. Coordination between medium-term generation planning and short-term operation in electricity markets. IEEE Trans Power Syst 2006;21(1):43-52.

[23] Shahidehpour M, Yamin H, Li Z. Market operations in electric power systems: forecasting, scheduling, and risk management. 1st ed. New York: Wiley-IEEE Press; 2002.

[24] Soares S, Lyra C, Tavares H. Optimal generation scheduling of hydrothermal power systems. IEEE Trans Power Ap Syst 1980:PAS-99(3):1107-18.

[25] Soares S, Salmazo C. Minimum loss predispatch model for hydroelectric power systems. IEEE Trans Power Syst 1997;12(3):1220-8.

[26] Wang Q, Wang J, Guan Y. Price-based unit commitment with wind power utilization constraints. IEEE Trans Power Syst 2013;28(3):2718-26.

[27] Wangensteen I, Botterud A, Flatabo N. Power system planning and operation in international markets-perspectives from the nordic region and Europe. Proc IEEE 2005;93(11):2049-59. 